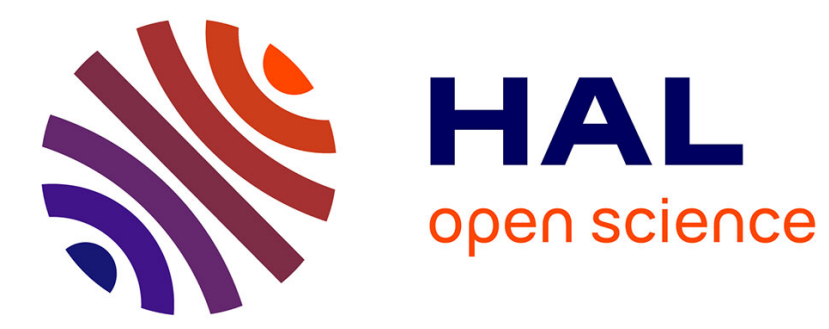

\title{
La gestion des retours de produits en fin de vie : le cas de la société IBM
}

\author{
Pauline Keh, Régis Meissonier, Florence Rodhain, Virigine Llorca
}

\section{To cite this version:}

Pauline Keh, Régis Meissonier, Florence Rodhain, Virigine Llorca. La gestion des retours de produits en fin de vie : le cas de la société IBM. Gestion - HEC Montréal, 2013, 38 (2), pp.88-96. 10.3917/riges.382.0088 . hal-01967280

\section{HAL Id: hal-01967280 \\ https://hal.science/hal-01967280}

Submitted on 30 Dec 2018

HAL is a multi-disciplinary open access archive for the deposit and dissemination of scientific research documents, whether they are published or not. The documents may come from teaching and research institutions in France or abroad, or from public or private research centers.
L'archive ouverte pluridisciplinaire HAL, est destinée au dépôt et à la diffusion de documents scientifiques de niveau recherche, publiés ou non, émanant des établissements d'enseignement et de recherche français ou étrangers, des laboratoires publics ou privés. 


\section{Chronique : Étude de cas}

\section{Les bénéfices de la gestion des retours de produits en fin de vie : le cas de la société IBM}

\section{Pauline Keh, Régis Meissonier, Florence Rodhain et Virginie Llorca}

Pauline Keh est doctorante en Sciences de Gestion au MRM-Cregor de l'Université de Montpellier 2, paulinekeh@gmail.com.

Régis Meissonier est professeur des Universités au MRM-Cregor de l'Université de Montpellier 2, regis.meissonier@univ-montp2.fr.

Florence Rodhain est maître de Conférences au MRM-Cregor de l'Université de Montpellier 2, florence.rodhain@univ-montp2.fr.

Virginie Llorca est Chef de projet développement \& transformation chez IBM France, virginie_llorca@fr.ibm.com. 


\section{Résumé}

Chaque année, de 20 à 50 millions de tonnes de déchets électroniques sont produits dans le monde et leur toxicité engendre de graves problèmes environnementaux et sanitaires. Cet article s'intéresse aux divers bénéfices liés à l'adoption d'une véritable gestion ou logistique des retours de produits en fin de vie. Tout d'abord, nous nous appuyons sur une étude de cas concernant l'usine d'IBM à Montpellier. Après avoir décrit le mode de gestion des retours de produits périmés et les conditions de succès qu'il respecte, nous listons les bénéfices variés qu'il entraîne, notamment les revenus supplémentaires, la réduction des dépenses, l'amélioration de la réputation de l'entreprise, le respect des lois et la création d'emplois locaux. Enfin, l'article insiste sur les implications de cette étude de cas pour les dirigeants, les consommateurs, les législateurs et la société en général.

Mots clés : logistique des retours, logistique inverse, déchets électroniques, enjeux, bénéfices, IBM, développement durable, responsabilité sociale

Fonctions : GRH, GOP, management 


\section{Chronique : Étude de cas}

\section{$\underline{\text { Les bénéfices de la gestion des retours de produits en fin de vie : le cas de la société IBM }}$ \\ Pauline Keh, Régis Meissonier, Florence Rodhain et Virginie Llorca}

Depuis plus de 30 ans, le secteur des équipements électriques et électroniques est en pleine expansion avec la création de nouveaux équipements dont les durées de vie et de service sont toujours plus courtes. Ainsi, on estime qu'il y a une production annuelle mondiale de 20 à 50 millions de tonnes de déchets électroniques dont la toxicité génère des risques pour l'environnement et la santé publique. Une façon de minimiser l'impact écologique et sociétal de ces déchets consiste à rendre plus responsables les producteurs en les incitant à prendre en charge la gestion de fin de vie de leurs équipements.

La «logistique des retours», ou «logistique inverse», est un processus qui permet aux fabricants de gérer les retours de la part de leurs clients de leurs produits arrivés en fin de vie ou en fin de service pour une revalorisation, une réutilisation ou un recyclage éventuels. Bien que la mise en œuvre d'un tel processus permette aux dirigeants de répondre aux attentes sociétales et législatives en matière de gestion de fin de vie des produits, on constate de nombreux freins à son implantation. En effet, le fait d'étendre la chaîne logistique afin d'y inclure des processus tels que la remise à neuf ou la remise en état (remanufacturing) ou encore la réutilisation complexifie la gestion des opérations et peut entraîner certaines difficultés. La logistique des retours fait alors l'objet d'un manque d'enthousiasme chez les décideurs qui, face à ces difficultés, ne perçoivent pas les atouts que comporte une meilleure gestion de fin de vie de leurs produits. Il vaut donc mieux faire connaître aux gestionnaires les bénéfices que cette logistique des retours peut apporter. C'est là l'objectif de cet article. En nous appuyant sur une étude de cas approfondie de l'usine d'IBM à Montpellier, nous nous interrogeons sur les solutions pouvant permettre de surmonter les barrières à l'implantation de la logistique des retours et sur les possibilités que cette activité est susceptible de représenter pour une entreprise. Par ailleurs, nous fondons nos propos sur d'autres écrits sur 
le sujet et sur d'autres exemples d'entreprises que nous avons pu relever dans la littérature. L'encadré 1 présente le cadre de recherche ainsi que la méthodologie utilisée.

Dans cet article, nous décrivons le modèle de logistique des retours mis en œuvre chez IBM à Montpellier ainsi que les conditions de son succès. Puis, nous faisons une synthèse des différentes possibilités qu'offre cette activité sur le plan économique. Par la suite, nous traitons des bénéfices de la logistique des retours sur le plan sociétal. Finalement, nous exposons les implications de cette étude pour les dirigeants, les consommateurs, les législateurs et la société en général.

\section{Encadré 1 - À propos de l'étude}

Nous avons recueilli des données au moyen de 41 entretiens menés entre 2009 et 2012 auprès de cadres dirigeants et intermédiaires de l'usine d'IBM à Montpellier, en France. Nous nous sommes aussi appuyés sur une observation faite sur le terrain et pendant notre participation à divers projets stratégiques et opérationnels.

\section{$\underline{\text { L'expérience d'IBM à Montpellier }}$}

Depuis 1995, l'usine d'IBM à Montpellier est responsable de la collecte et du traitement des retours pour

toutes les entités d'IBM en Europe. À ce titre, deux types de flux reviennent à Montpellier : les retours de pièces et les retours de serveurs. Les pièces peuvent revenir à la suite d'une panne subie par la clientèle, d'un changement de configuration ou en raison de surplus ou d'obsolescence dans les stocks d'IBM. Quant aux serveurs, ils reviennent à la suite d'une fin de contrat de location ou d'un renouvellement de contrat entraînant la vente du modèle supérieur contre le rachat de l'ancien.

\section{Les modes de gestion des retours de produits usagés}

Une fois récupérés et acheminés jusqu'à Montpellier, les produits usagés peuvent être retraités à travers les processus suivants. 
La remise à neuf. Lorsque des serveurs reviennent de la clientèle en raison d'une fin de contrat, ils sont testés et, en fonction de la demande des clients, ils pourront suivre le processus de remise à neuf. Les appareils réusinés sont entièrement composés de pièces et de modules usagés, puis ils sont revendus sur le marché des produits d'occasion en tant qu'appareils certifiés IBM, mais sans garantie. D'autres appareils, dits «hybrides», comprennent à la fois des pièces usagées et des pièces neuves et sont revendus avec le même contrat et les mêmes garanties qu'un appareil neuf. Les serveurs en mauvais état, ou ceux pour lesquels il n'y a plus de besoin, sont redirigés vers le processus de recyclage.

La réutilisation. Lorsque des pièces conformes reviennent de la clientèle par suite d'un changement de configuration, elles peuvent, en fonction de leur valeur et des besoins mondiaux de la production, être réutilisées à l'interne de deux façons. Il peut s'agir d'une réutilisation sur le marché des produits neufs : les pièces sont retravaillées à l'interne ou à l'externe, testées, nettoyées, puis réétiquetées, pour enfin revenir en stock en tant que pièces équivalentes à des pièces neuves. Elles peuvent alors être réutilisées par la production d'IBM de serveurs neufs. Il peut également d'agir d'une réutilisation sur le marché des produits d'occasion : les pièces usagées pourront également être réutilisées telles quelles par la production de serveurs d'occasion. Les pièces à faible valeur ou celles pour lesquelles il n'y a plus de besoin sont redirigées vers le processus de recyclage.

La réparation. On teste les pièces défectueuses qui reviennent de la clientèle afin de déterminer l'origine de la panne, puis elles sont réparées chez le fabricant d'origine avant d'être renvoyées aux centres de maintenance d'IBM. Les pièces non réparables sont redirigées vers le processus de recyclage.

Le recyclage. Les produits usagés qui ne se prêtent pas aux processus de retraitement décrits précédemment sont démantelés et peuvent ensuite être revalorisés de trois manières : par la revente de 
pièces détachées aux intermédiaires, par la revalorisation des métaux précieux ou par le recyclage des matières premières.

L'élimination. Certains matériaux ou produits chimiques non recyclables sont éliminés par incinération avec une valorisation énergétique. Aucun déchet en provenance de l'usine d'IBM à Montpellier n'est envoyé dans une décharge publique.

\section{$\underline{\text { Les conditions de succès respectées par l'usine d'IBM à Montpellier }}$}

\section{Intégrer les retours à la chaîne logistique existante}

En 1989, lorsque IBM a mis en place un programme de récupération des produits usagés en Europe, elle n'a pas tout de suite opté pour une gestion centralisée des retours. La majorité des pays européens disposait alors d'un centre IBM responsable de la gestion de retours au niveau national. Chacun de ces centres possédait ses propres ressources, infrastructures et partenariats nécessaires à la gestion et à l'élimination des produits usagés. Dans le milieu des années 1990, IBM a décidé de centraliser tous les retours européens à un seul emplacement, celui de Montpellier. Ce choix a permis non seulement de gérer un seul stock de pièces et de machines usagées en Europe, et donc d'optimiser les possibilités de réutilisation et de revente, mais également d'effectuer de nombreuses synergies en matière d'infrastructures et de ressources.

Mutualiser les infrastructures. L'entité d'IBM à Montpellier étant à l'origine un lieu de production de serveurs neufs, elle disposait déjà des installations et des équipements permettant d'entreposer, d'assembler et de tester les serveurs neufs. Ainsi, des synergies ont pu être opérées et la logistique des retours partage aujourd'hui les mêmes ateliers d'assemblage, salles de test et infrastructures d'entreposage que la chaîne logistique traditionnelle. En outre, depuis plus de 15 ans, IBM sous-traite ses activités de transport, de réception et de magasinage à un prestataire logistique. Aujourd'hui, cette société gère pour le compte d'IBM les flux logistiques aussi bien à l'aller qu'au retour. Qu'ils soient neufs ou usagés, les 
produits sont amenés à partager les mêmes modes de transport ainsi que les mêmes infrastructures d'entreposage.

Mutualiser les ressources. De la même façon, lorsque l'activité de logistique des retours a débuté à Montpellier dans les années 1990, l'emplacement avait déjà les ressources et les compétences nécessaires aux différentes opérations de la chaîne logistique classique. Le choix d'implanter l'activité de logistique des retours à Montpellier a permis de réaliser des synergies et de limiter ainsi les investissements indispensables en matière de ressources humaines et matérielles. Une même équipe gère avec les mêmes outils de production l'assemblage et le test des serveurs neufs et d'occasion. De même, les personnes chargées de la finance, de l'ingénierie, du contrôle de gestion et du contrôle de production pour les machines et pièces neuves gèrent également ces fonctions pour les machines et pièces usagées.

Une recherche menée auprès de deux sociétés ${ }^{1}$ a montré qu'un facteur déterminant de l'efficacité et de la viabilité du système de logistique des retours est la capacité des entreprises de réutiliser les infrastructures, le personnel et les équipements existants, car les coûts d'acquisition de nouvelles installations ainsi que de nouveaux équipements de production et de manutention peuvent être prohibitifs.

De plus, l'utilisation du personnel actuel est essentielle à la viabilité de la logistique des retours étant donné qu'elle permet de minimiser les coûts supplémentaires d'embauche et de formation.

\section{$\underline{\text { La réutilisation des infrastructures, du personnel et des équipements existants comme facteur de }}$} succès

\section{Intégrer les flux et les opérations des produits usagés aux systèmes d'information existants}

La mise en œuvre du processus de logistique des retours à Montpellier a été accompagnée d'investissements dans les systèmes d'information afin que les outils existants puissent être réutilisés et intègrent la gestion des flux tant à l'aller qu'au retour. Ainsi, l'outil de planification, de production ou 
encore le progiciel de gestion intégré (ERP) gère à la fois la logistique aller et la logistique retour et offre ainsi une vision globale de la chaîne d'approvisionnement.

Afin d'optimiser la réutilisation, l'usine d'IBM à Montpellier intègre les retours de pièces dans son système de planification, et la couverture totale du calcul des besoins comprend non seulement le stock de pièces déjà remises à neuf, mais également trois niveaux de retours prévisionnels, soit les prévisions des inventaires à court, à moyen et à long terme.

Les prévisions à court terme s'appuient sur les pièces déjà revenues à l'usine mais pas encore remises à neuf. Cet inventaire est pris en compte à $100 \%$ dans la couverture des besoins. Les prévisions à moyen terme se basent sur les mises à niveau déjà expédiées chez les clients pour lesquelles les retours associés sont connus mais pas encore réceptionnés à l'usine. Il apparaît que $80 \%$ de cet inventaire est intégré à la couverture, ce qui laisse $20 \%$ d'incertitude sur la qualité ou la quantité finale de ces retours. Enfin, les prévisions à long terme se fondent sur les planifications d'expédition des mises à niveau. Ici, seulement 50 $\%$ de l'inventaire est intégré à la couverture, ce qui laisse $50 \%$ d'incertitude quant aux volumes réels expédiés et à la qualité ou à la quantité finale des retours.

L'intégration de ces trois niveaux de retours prévisionnels dans le calcul des besoins offre à IBM la possibilité d'anticiper au maximum ces retours et de privilégier au mieux la réutilisation avant l'achat et la fabrication de composants neufs. De plus, IBM a mis au point des systèmes d'information qui permettent de prendre en compte les besoins de toutes les usines d'IBM dans le monde et de comparer ces besoins avec tous les stocks de pièces réutilisables. Ainsi, des pièces remises à neuf à l'usine de Montpellier peuvent ensuite être envoyées à celle de Singapour pour les besoins de sa production. Notre étude rejoint ainsi les travaux d'autres chercheurs qui considèrent que le rôle des technologies de l'information et de la communication est déterminant dans la gestion des retours, puisque celles-ci permettent de suivre les produits, de prévoir les retours et d'optimiser ainsi la gestion des inventaires ${ }^{2}$. 


\section{$\underline{\text { Les atouts de la gestion des retours des produits en fin de vie pour les entreprises }}$}

La gestion des retours des produits en fin de vie permet à l'entreprise d'accroitre ses revenus en accédant au marché des produits d'occasion, de générer des revenus en revalorisant des pièces et des composants, de diminuer les achats de pièces et de composants neufs, de réduire les taxes et frais environnementaux et, enfin, d'améliorer sa réputation.

\section{Accroître les revenus en accédant au marché des produits d'occasion}

En récupérant et en retravaillant les produits ou composants usagés, les fabricants peuvent accéder à ce nouveau marché en pleine croissance et créer ainsi de nouvelles sources de revenus et de profits ${ }^{3}$. Selon une étude du groupe Xerfi parue en 2008, le marché total des produits d'occasion s'élevait à 5 milliards d'euros en 2007 (sauf l'automobile, les antiquités, les œuvres d'art et l'immobilier) et connaît un taux de croissance de $6 \%$ chaque année (voir l'encadré 2). Ainsi, «les pièces réusinées offrent la possibilité de segmenter le marché et de satisfaire différents besoins des clients en proposant une plus large gamme d'offres ${ }^{4} \gg$. Notre étude révèle que, à partir des retours de serveurs et de pièces usagés, l'usine de Montpellier a été en mesure de proposer au marché européen deux types d'appareils retravaillés qui permettent à IBM de mieux s'adapter aux besoins et aux budgets des différents clients et d'élargir ainsi son marché. Aujourd'hui, dans le domaine des grands serveurs, plus de $35 \%$ des appareils IBM vendus sont des appareils hybrides ou réusinés.

\section{Encadré 2 - La réutilisation et le retraitement des produits usagés : le cas de la société Caterpillar}

Les produits remis à neuf peuvent coûter, en moyenne, la moitié moins cher que les produits neufs, ce marché pouvant être extrêmement rentable. Chez Caterpillar, les marges des opérations de remise à neuf sont si impressionnantes que la société a fait de cette activité une division séparée. L'unité a réalisé des ventes de plus de 1 milliard de dollars en 2005 et devrait croître de $15 \%$ par an durant les prochaines 
années. Ce n'est qu'une petite part des 36 milliards de dollars de chiffre d'affaires mondial du géant spécialiste des équipements lourds, mais la remise à neuf est l'une des divisions de Caterpillar qui enregistrent la plus forte croissance.

Source : www.businessweek.com/stories/2006-09-24/everything-old-is-new-again; traduction libre.

\section{Générer des revenus en revalorisant des pièces et des composants}

Avant d'être éliminées, certaines pièces, qui ne sont pas destinées à une réutilisation interne, sont revendues sur le marché des produits d'occasion par des intermédiaires. Enfin, celles qui ne sont pas revendues sont démantelées et les métaux précieux que contiennent certains composants (or, argent, palladium ou cuivre) sont revalorisés auprès d'éliminateurs spécialisés. Le recyclage des pièces et composants génère des revenus de plus de deux millions d'euros par an, ce qui permet à l'usine de Montpellier de recouvrer entièrement les coûts de démantèlement, de tri et de recyclage.

\section{Diminuer les achats de pièces et composants neufs}

Certaines pièces et certains composants qui reviennent de la clientèle peuvent être retravaillés, voire remis à neuf et ainsi être réintégrés dans la chaîne de production. La réutilisation de ces pièces permet de réduire l'achat et la fabrication de composants neufs et génère des économies pour l'entreprise. Prenons l'exemple des processeurs XY, le cœur des grands serveurs IBM, qui comptent parmi les éléments les plus chers de ces grands systèmes. En 2009, 72 de ces processeurs sont revenus à Montpellier après un changement de configuration chez des clients. En parallèle, l'usine de Dublin, en Irlande, avait besoin de 100 processeurs XY pour assurer sa production de serveurs neufs pour l'année 2009. Ces 72 processeurs ont été intégrés dans la couverture lors du calcul des besoins, et après avoir été remis à neuf à Montpellier, ils ont été fournis à Dublin tout au long de l'année. En 2009, pour des besoins de 100 processeurs XY, l'usine de Dublin en a réutilisé 72 en provenance de Montpellier et n'en a acheté que 28 neufs, ce qui a permis à l'entreprise d'économiser jusqu'à plusieurs millions d'euros annuellement. 


\section{Réduire les taxes et frais environnementaux}

Il est souvent considéré que, pour une entreprise, le fait d'améliorer la protection de l'environnement engendre automatiquement des coûts supplémentaires. Cependant, depuis le milieu des années 1990, ce constat est contesté par des travaux qui estiment, au contraire, qu'en améliorant sa performance environnementale une entreprise aura tendance à améliorer sa performance financière ${ }^{5}$. Ces travaux rejoignent l'hypothèse selon laquelle la pollution est une forme de gaspillage économique, puisqu'elle résulte d'une utilisation inefficace des ressources ${ }^{6}$. Enfin, certains auteurs ajoutent qu'une meilleure performance environnementale entraîne une diminution des taxes et frais environnementaux, car elle permet, d'une part, d'éviter les amendes et les litiges et, d'autre part, de diminuer les taxes liées à l'élimination et à la mise en décharge des produits ${ }^{7}$. Dès la fin des années 1990, les sociétés Black \& Decker et Xerox ont décidé d'être des entreprises sans déchets ou de ne rien envoyer dans les décharges publiques, ce qui leur a vite permis d'économiser des millions de dollars de frais de mise en décharge ${ }^{8}$.

\section{Améliorer la réputation de l'entreprise}

De plus en plus de clients sont sensibles à la problématique environnementale, et «il devient de plus en plus fréquent que les administrations publiques incluent la performance environnementale dans leurs critères de choix des fournisseurs de biens ou de services ${ }^{9} »$. Le fait de récupérer les produits, de les réutiliser ou d'en assurer une élimination responsable peut permettre à une entreprise d'accroître sa réputation en matière de respect de l'environnement et ainsi d'augmenter la fidélité de ses clients, voire d'en attirer de nouveaux. Dans notre étude de cas, nous avons observé que la logistique des retours était un moyen pour IBM de rester cohérente avec l'image d'entreprise «verte» que la multinationale cultive depuis de nombreuses années. En effet, l'environnement fait partie des valeurs essentielles véhiculées par IBM et contribue fortement à son image de marque. La gestion des produits en fin de vie est donc naturellement une problématique importante pour la multinationale, dans la mesure où sa réputation supporterait mal la présence de matériel dans des décharges, en Asie par exemple. Ainsi, dès 1989, IBM a mis en place un programme de récupération de ses produits usagés avec comme objectif d'optimiser la 
réutilisation ou le recyclage des produits en fin de vie et de diminuer au maximum le volume de déchets envoyé dans les décharges. Un responsable en matière d'environnement d'IBM précise que «l'emplacement de Montpellier n'envoie aucun déchet dans les décharges publiques. Il s'est pour cela entouré d'une vaste filière de recycleurs spécialisés dans le verre, le plastique, le papier et les métaux ferreux. Cette filière de recyclage a été choisie par IBM et certifiée selon des critères environnementaux en accord avec les exigences légales.»

\section{$\underline{\text { Les bénéfices de la logistique des retours }}$}

La logistique des retours permet d'anticiper les exigences légales ou de répondre à celles-ci, de réduire la consommation d'énergie et de ressources naturelles ainsi que de créer ou de préserver des emplois locaux.

\section{Anticiper les exigences légales ou y répondre}

Depuis le début des années 1990, des directives sont adoptées dans différents pays afin de minimiser l'impact environnemental des déchets électroniques. Bien que plus ou moins strictes selon les gouvernements, ces directives tendent toutes vers un contrôle renforcé des méthodes de gestion des déchets en matière de transport, de retraitement ou d'élimination. En Europe, par exemple, la directive DEEE (2002/96/EC) de 2005 rend le producteur responsable de la collecte et du recyclage des produits électriques et électroniques en fin de vie. En parallèle, la directive européenne RoHS (2002/95/EC) de 2006 impose aux fabricants de supprimer l'utilisation de certaines substances dangereuses telles que le plomb, le cadmium et le mercure. Au début des années 1990, soit 15 ans avant l'entrée en vigueur de ces deux directives, IBM a commencé à interdire l'utilisation dans ses produits de certaines substances dangereuses comme le cadmium et a mis en place une solution de logistique des retours pour la collecte et le traitement du matériel informatique en fin de vie. Ces initiatives ont permis à l'entreprise de devancer ces deux directives européennes et d'être en conformité avec elles avant même qu'elles n'entrent en vigueur. 
Si aujourd'hui certains pays continuent d'exporter leurs déchets vers les pays en voie de développement, on peut penser qu'il existe une tendance à une évolution vers un plus grand contrôle et vers des mesures environnementales au moins aussi strictes que celles instaurées en Europe. Ainsi, une démarche environnementale est en train de s'imposer à l'ensemble des fabricants d'équipements électriques et électroniques, qui, tôt ou tard, devront se conformer à des lois concernant la gestion de leurs produits en fin de vie. La logistique des retours représente donc un moyen de répondre à ces pressions réglementaires, voire de les anticiper. En effet, de nombreux auteurs croient que les entreprises qui adoptent des stratégies environnementales proactives, c'est-à-dire qui dépassent volontairement les exigences réglementaires, peuvent bénéficier de l'avantage du pionnier et éventuellement influencer l'évolution des législations ${ }^{10}$.

\section{Réduire la consommation d'énergie et de ressources naturelles}

En favorisant la réutilisation des pièces et composants usagés, la logistique des retours permet d'allonger le cycle de vie des produits, de diminuer l'achat et la fabrication de pièces neuves et donc de réduire les besoins en ressources naturelles. Une étude montre que les processus de retraitement tels que la remise à neuf sont beaucoup moins énergivores que la fabrication de produits neufs et que, en plus d'allonger le cycle de vie du produit, ils permettent de réduire considérablement la consommation d'énergie ${ }^{11}$.

«En termes de consommation d'énergie, la remise en état d'un produit ne nécessite qu'environ 15 \% de l'énergie utilisée pour fabriquer le même produit neuf. Au niveau mondial, les économies d'énergie faites en favorisant la remise à neuf plutôt que la fabrication d'un produit neuf sont estimées à 400 trillions de BTU par an, ce qui est considérable. Afin de mettre ce chiffre en perspective, disons qu'il équivaut à 16 millions de barils de pétrole brut (environ 350 camionsciternes), ou à une quantité d'essence pouvant faire fonctionner 6 millions de voitures pendant un an. [...] Sur la base de l'estimation de ces 400 trillions de BTU économisés chaque année, la remise à neuf évite la génération d'environ 28 millions de tonnes de $\mathrm{CO}_{2}$ par an, soit l'équivalent de 10 centrales électriques de 500 mégawatts alimentées au charbon ${ }^{12}$.» 


\section{Créer ou préserver des emplois locaux}

Des auteurs sont aussi d'avis que la remise à neuf s'avère une industrie «nationale», puisque, aux ÉtatsUnis, la majorité des produits usagés sont récoltés, triés, retravaillés puis reconsommés localement : «l'industrie de la remise à neuf a tendance à rester à la maison; c'est une industrie nationale qui favorise l'emploi et la formation locale ${ }^{13} \gg$.

Notre étude de cas de l'usine d'IBM à Montpellier révèle qu'un des atouts majeurs de l'activité de logistique des retours est de générer des revenus afin de justifier la préservation des emplois dans cette usine. En effet, depuis le début des années 1990, face à une stratégie globale de centralisation et de rationalisation de la production, cet emplacement a vu son activité initiale de fabrication de serveurs diminuer au profit de pays plus attrayants quant à la main-d'œuvre ou à la fiscalité. Afín de compenser cette baisse d'activité et de sauvegarder ses effectifs, l'usine s'est lancée dans une transformation de l'emplacement dont l'objectif était de trouver de nouvelles missions entraînant des revenus et justifiant ainsi la préservation des emplois locaux. Comme l'explique un responsable, «depuis une quinzaine d'années, la logistique des retours a permis à l'usine d'accompagner la baisse d'activité de production de serveurs neufs jusqu'à aujourd'hui, de reconvertir et donc de sauvegarder de $25 \%$ à $30 \%$ des effectifs qui travaillaient en production dans le début des années 1990». Ainsi, en 1993, l'effectif total de l'usine était dédié à la production, alors qu'aujourd'hui plus de la moitié des ressources sont rentabilisées par les revenus générés par ces nouvelles missions, dont un quart par la logistique des retours. Selon François Augnet, vice-président de TRW Automotive Europe et Asie-Pacifique, «la remise à neuf présente une très bonne occasion pour l'Europe de conserver et de créer des emplois. À l'heure où de nombreuses entreprises délocalisent les emplois hors de l'Union européenne, les entreprises qui œuvrent dans la remise à neuf n'ont aucun intérêt à faire de même compte tenu des coûts logistiques qu'occasionneraient de telles opérations ${ }^{14} »$.

\section{Les implications de la logistique des retours}


Nous verrons ici les implications qu'a la logistique des retours pour les dirigeants d'entreprise, les consommateurs, les législateurs et la société.

\section{Les implications pour les dirigeants d'entreprise}

Si de nombreux fabricants de matériel électronique sont encore réticents à l'idée de mettre en œuvre un système de logistique des retours, il est probable que la montée en puissance des pressions sociétales et légales ne leur laissera bientôt plus le choix. La logistique des retours comprend des processus nouveaux et complexes, et il est important que les producteurs adoptent une stratégie proactive et réfléchissent dès à présent à une solution optimale : où implanter les centres de retraitement? Quelles options de retraitement privilégier? Quels partenariats choisir?

L'étude de cas de l'usine d'IBM à Montpellier, appuyée par d'autres exemples d'entreprises, indique que l'efficacité et la rentabilité d'un système de logistique des retours dépendent largement du degré de réutilisation des ressources et des compétences existantes. En effet, afin de réduire les investissements nécessaires ainsi que les difficultés opérationnelles liées à la mise en œuvre de la logistique des retours, il est conseillé d'intégrer la gestion des retours à la gestion de la chaîne logistique classique. L'entreprise évitera ainsi de dédoubler les ressources nécessaires en réutilisant au maximum les installations, les équipements de même que les compétences et les systèmes d'information existants.

\section{Les implications pour les consommateurs}

En proposant au client de récupérer les produits qui ne répondent plus à ses besoins et en lui laissant le choix entre des produits neufs, à la fine pointe de la technologie, et des produits de génération plus ancienne, retravaillés et $30 \%$ à $40 \%$ moins chers, la logistique des retours représente un moyen d'améliorer le service à la clientèle. Elle permet ainsi de replacer le client au centre des préoccupations de l'entreprise et de contrebalancer l'évolution du marketing depuis les années 1990, qui tend à se concentrer sur l'efficience et le rendement au détriment de la prestation de service ${ }^{15}$. Cependant, il convient de garder 
quelques réserves par rapport à ces observations, car s'il est vrai que la remise à neuf permet aux consommateurs de trouver des produits moins chers, on peut se demander s'il ne s'agirait pas d'une nouvelle subtilité de la logique du marketing actuelle qui pousse par tous les moyens à la consommation sans se soucier réellement des besoins des clients : en offrant un produit moins cher, l'objectif premier de la remise à neuf est-il réellement de faire faire des économies aux consommateurs ou est-il plutôt de susciter de nouveaux besoins chez de nouveaux consommateurs?

\section{Les implications pour les législateurs}

S'il a été démontré que divers intérêts économiques ou sociétaux peuvent motiver les dirigeants à mettre en œuvre une logistique des retours, il n'en reste pas moins que la régulation fait partie des motivations principales. Ainsi, sans aller jusqu'à partager l'hypothèse de Michael Porter selon laquelle une forte régulation stimule l'innovation et améliore la performance des entreprises, nous pouvons avancer qu'une forte régulation incite les entreprises à s'intéresser à la fin de vie de leurs produits et à implanter les moyens de s'y conformer. Les directives DEEE et RoHS sont souvent citées pour leur impact positif sur le comportement des fabricants de matériel électronique européens, mais au niveau international les directives sont rares et de nombreux pays continuent d'exporter clandestinement leurs déchets vers l'Asie ou l'Afrique. Ainsi, à l'échelle internationale, il existe un seul traité, soit la convention de Bâle, qui vise à minimiser les mouvements clandestins de déchets dangereux et qui exige que chaque expédition soit précédée d'une notification au pays importateur et soumise à son consentement. Cette convention, signée par 179 pays, est entrée en vigueur en 1992. En 1995, le Ban Amendment a proposé de renforcer cette convention en interdisant l'exportation des déchets dangereux d'un pays membre de l'Organisation de coopération et de développement économiques (OCDE) vers un pays non membre. Aujourd'hui, certains

pays influents tels que les États-Unis, le Canada, l'Australie et le Japon refusent de ratifier cet amendement. La problématique de la gestion de fin de vie des produits électroniques souffre donc d'un manque d'encadrement légal dans certains pays et d'une absence d'entente et de coordination au niveau international. 


\section{Les implications pour la société}

Une première implication sociétale mise en avant par notre étude est l'occasion que la logistique des retours présente en termes d'emplois, et plus particulièrement d'emplois locaux. En effet, il apparaît que, dans le cadre de la gestion de produits usagés, une certaine proximité avec la source des retours est parfois indispensable au vu des législations en vigueur et des coûts logistiques engendrés par une telle activité. La logistique des retours semble donc être une industrie «nationale» qui pourrait présenter des possibilités d'emploi et de formation locaux dans les pays industrialisés mais également dans les pays en voie de développement. En effet, des pays comme la Chine et le Brésil génèrent d'ores et déjà des volumes de déchets électroniques importants et les occasions de revalorisation commencent à attirer certains industriels. C'est le cas d'IBM, qui a annoncé en 2012 l'ouverture de son premier centre de remise à neuf à Shenzhen, en Chine. Selon Richard Dicks, directeur général d'IBM Global Asset Recovery Services, la Chine est un marché prometteur, car «la demande de produits de technologies de l'information sur les marchés émergents est en pleine croissance, et toutes les entreprises ne veulent pas nécessairement acheter des produits nouveaux. [...] Cette usine de Shenzhen permettra aux clients d'acquérir un équipement d'occasion à des prix abordables tout en participant à la réduction de l'impact des déchets électroniques sur l'environnement grâce à la réutilisation d'appareils qui, autrement, auraient fini dans les décharges ${ }^{16} . »$

Une deuxième conséquence sociétale de la logistique des retours, fréquemment soulignée par les chercheurs et les industriels, est l'allongement du cycle de vie des produits ainsi que la réduction de la consommation d'énergie et de ressources naturelles. Ainsi, selon certains auteurs, la logistique des retours pourrait permettre aux entreprises de répondre aux demandes croissantes de responsabilité sociale et d'atteindre plus globalement les objectifs sociaux visant à modifier la tendance actuelle de surconsommation des ressources $^{17}$. Il faut toutefois rester prudent par rapport à ces postulats. En effet, peut-on véritablement parler de changement dans la manière de consommer? L'activité de remise à neuf 
n'aurait-elle pas un effet boomerang? Car s'il est vrai que les activités de retraitement utilisent moins de ressources, il reste qu'elles s'adressent à de nouveaux clients et amènent un élargissement du marché. La demande étant plus importante, les volumes de production augmentent et, au final, la consommation d'énergie et de ressources naturelles augmente également. La logistique des retours ne résout pas un problème sur le plan de la logique de production. Une solution socialement responsable repose aussi sur une consommation moindre, de même que sur une production moindre, où l'on fabrique des produits qui durent plus longtemps, plutôt que de proposer chaque année de nouveaux produits qui rendent obsolètes les générations précédentes afin de stimuler d'autres achats. Certes, la responsabilisation des entreprises dans la gestion de la fin de vie de leurs produits s'avère une belle avancée. Toutefois, il importe aussi d'opérer en amont, c'est-à-dire dans le design et la conception du produit.

\section{$\underline{\text { Mot de la fin }}$}

Cet article a mis en évidence différents atouts de la logistique des retours susceptibles de motiver la mise en œuvre d'une telle activité. Tout d'abord, cette activité permet aux industriels de générer des revenus supplémentaires sur le marché des produits d'occasion, de minimiser leurs dépenses en réutilisant une partie des pièces et composants usagés et d'améliorer leur image et leur réputation. Elle permet également aux industriels d'intégrer certaines exigences écologiques et sociétales en matière de gestion des déchets. Enfin, elle peut être une source de création ou de préservation d'emplois. Que ce soit pour des raisons économiques, légales ou stratégiques, les fabricants d'équipements électriques et électroniques ont ainsi intérêt à porter une attention particulière à la gestion de leurs produits en fin de vie et à considérer les retours comme une occasion de générer de la valeur, et non comme de simples déchets dont la manipulation est contraignante et coûteuse.

\section{$\underline{\text { Références }}$}

Ambec, S., Lanoie, P. (2007), «When and why does it pay to be green?», CIRANO Burgundy Reports, $\mathrm{n}^{\circ}$ 2007rb-03. 
Ambec, S., Lanoie, P. (2008), «Does it pay to be green? A systematic overview», The Academy of Management Perspectives, vol. 22, $\mathrm{n}^{\circ}$ 4, p. 45-62.

Andel, T. (1997), «Reverse logistics: A second chance to profit», Transportation \& Distribution, vol. 38, $\mathrm{n}^{\circ}$ 7, p. 61-66.

Aylott, C. (2012), «IBM opens first server remanufacturing center in China», Emerging Money, http://emergingmoney.com/china/ibm-opens-first-server-remanufacturing-center-in-china-ibm/.

Chouinard, M., D'Amours, S., Ait-Kadi, D. (2005), «Integration of reverse logistics activities within a supply chain information system», Computers in Industry, vol. 56, n 1, p. 105-124.

De Brito, M.P., Dekker, R., Flapper, S.D.P. (2005), «Reverse logistics: A review of case studies», dans Fleischmann, B., Klose, A. (dir.), Distribution Logistics Advanced Solutions to Practical Problems, Springer-Verlag, p. 243-281.

Dowlatshahi, S. (2000), «Developing a theory of reverse logistics», Interfaces, vol. 30, n 3, p. 143-155.

Dowlatshahi, S. (2005), «A strategic framework for the design and implementation of remanufacturing operations in reverse logistics», International Journal of Production Research, vol. 43, $\mathrm{n}^{\circ} 16, \mathrm{p}$. 3455-3480.

Giuntini, R., Gaudette, K. (2003), «Remanufacturing: The next great opportunity for boosting US productivity», Business Horizons, novembre-décembre, p. 41-48.

Hart, S.L. (1997), «Beyond greening: Strategies for a sustainable world», Harvard Business Review, vol. $75, \mathrm{n}^{\circ} 1, \mathrm{p} .66-76$.

Jayaraman, V., Guide, V.D.R., Srivastava, R. (1999), «A closed-loop logistics model for remanufacturing», Journal of the Operational Research Society, vol. 50, n 5, p. 497-508.

Kumar, S., Putnam, V. (2008), «Cradle to cradle: Reverse logistics strategies and opportunities across three industry sectors», International Journal of Production Economics, vol. 115, n 2, p. 305315.

Lankoski, L. (2006), «Environmental and economic performance: The basic links», dans Schaltegger, S., Wagner, M. (dir.), Managing the Business Case for Sustainability, Greenleaf Publishing, p. 3246.

Lund, R.T., Hauser, W.M. (2010), «Remanufacturing: An American perspective», www.reman.org/Papers/ICRM \%20APRA \%20version.pdf.

Maslennikova, I., Foley, D. (2000), «Xerox’s approach to sustainability», Interfaces, vol. 30, n 3, p. 226233.

Monnet, M. (2006), «L'élaboration des stratégies de logistique inversée», Gestion, vol. 31, n 3, p. 78-84.

Paulin, M. (2009), «La relation client hors service?», Gestion, vol. 33, n 4, p. 41-47.

Porter, M.E., Van der Linde, C. (1995), «Green and competitive: Ending the stalemate», Harvard Business Review, vol. 73, n 5, p. 120-134. 
Rahman, S., Subramanian, N. (2012), «Factors for implementing end-of-life computer recycling operations in reverse supply chains», International Journal of Production Economics, vol. 140, $\mathrm{n}^{\circ} 1$, p. $239-248$.

ReMaTecNews (2010), «An industry with huge potential», ReMaTecNews, vol. 10, n 4, p. 10-13.

Seitz, M., Wells, P. (2005), «Business models and closed-loop supply chains: A typology», Supply Chain Management, vol. 10, $\mathrm{n}^{\circ} 3-4$, p. 249-251.

\section{$\underline{\text { Note }}$}

${ }^{1}$ Dowlatshahi (2005).

${ }^{2}$ Chouinard et al. (2005), De Brito et al. (2005), Rahman et Subramanian (2012).

${ }^{3}$ Dowlatshahi (2000), Jayaraman et al. (1999).

${ }^{4}$ Kumar et Putnam (2008 : 313; traduction libre).

${ }^{5}$ Ambec et Lanoie (2007: 12).

${ }^{6}$ Porter et Van der Linde (1995).

${ }^{7}$ Ambec et Lanoie (2008), Lankoski (2006).

${ }^{8}$ Andel (1997), Maslennikova et Foley (2000).

${ }^{9}$ Ambec et Lanoie (2008 : 47; traduction libre).

${ }^{10}$ Ambec et Lanoie (2007, 2008), Monnet (2006).

${ }^{11}$ Giuntini et Gaudette (2003).

12 Giuntini et Gaudette (2003 : 44; traduction libre).

${ }^{13}$ Lund et Hauser (2010:6; traduction libre).

${ }^{14}$ ReMaTecNews (2010 : 12-13; traduction libre).

${ }^{15}$ Paulin (2009).

${ }^{16}$ Aylott (2012; traduction libre).

${ }^{17}$ Hart (1997), Seitz et Wells (2005). 